$19^{\text {th }}$ European Symposium on Computer Aided Process Engineering - ESCAPE19

J. Jeżowski and J. Thullie (Editors)

(C) 2009 Elsevier B.V./Ltd. All rights reserved.

\title{
Adaptation and testing of data reconciliation software for CAPE-OPEN compliance
}

\author{
Eric Radermecker, ${ }^{a}$ Marie-Noëlle Dumont, ${ }^{\mathrm{b}}$ Georges Heyen ${ }^{\mathrm{a}, \mathrm{b}}$ \\ ${ }^{a}$ BELSIM s.a., Rue Georges Berotte 24a, 4470 Saint-Georges-sur-Meuse, Belgium \\ ${ }^{b}$ Université de Liège, Chemical engineering, Sart Tilman B6, 4000 Liège, Belgium, \\ mn.dumont@ulg.ac.be
}

\begin{abstract}
The experience gained in the development of a CAPE-OPEN 1.0 thermo socket for the BELSIM-VALI software is presented. VALI is a data validation and reconciliation software that provides consistent mass and energy balances, reliable and accurate Key Performance Indicators and soft sensors. It is not a simulation software per definition, but rather a powerful equation based software that is used online, to monitor and manage the operations of different processes.

VALI has its own thermodynamic database of chemical compounds (> 800 fluids, > 650 solids) and methods. After demands of our clients it was decided to make VALI compliant with other thermodynamic packages via a CAPEOPEN 1.0 thermo socket. The source code of the VALI physical property modules had to be modified, to call the Material Object components instead of built-in thermodynamic functions (thermo socket).

In the user interface, we had to develop a CAPE-OPEN thermo plugs viewer to allow the selection of a CAPE-OPEN thermo plug from our Graphical User Interface (GUI).

Several case studies were analysed, with performance comparison between the native thermodynamic model, and properties obtained from several CAPEOPEN thermo plugs. We will particularly analyse here the modelling of a gas liquefaction system and a distillation case study.
\end{abstract}




\section{Keywords: Cape-Open, physical properties, data reconciliation}

\section{Introduction}

VALI is an advanced data validation and reconciliation software, used online in industry to improve the accuracy of plant data. It is not a simulation software and although process simulation and advanced data validation and reconciliation are similar on several aspects, they are quite different as well. The main difference being the way plant data are handled. In simulation, plant data are used to tune various parameters of simulation models, giving a better model, while in data validation plant data are used to check and correct measurements, giving better information of the actual state of the plant.

Both process simulation and data validation and reconciliation are based on a mathematical model of a process. These models are built using predefined objects modeling the process units operations and the products that are handled. Some information must be available about the physical properties of the chemical components present in the system like their molecular weight, their density, etc.

On the mathematical side, process simulation has been traditionally developed on a modular sequential approach. Each process unit is represented by a mathematical model that calculates the state of the output streams knowing the state of the input streams and some unit operation specifications. Each unit is modeled after the other. When some streams are recycled, convergence algorithms are to be used.

Using such an approach is not possible for data validation and reconciliation. Instead the program must generate equations that relate inputs and outputs. An optimization algorithm is then used to find the set of values that are as close as possible to the measurements while satisfying the equations.

\section{Learning Phase}

As a first step of this work, it was important to have a better understanding of both the CAPE-OPEN standards and the COM technology, before even starting the development work. A training course was followed, given by Prosim that has a long experience in implementations of CAPE-OPEN standards.

\subsection{COM technology}

COM (Component Object Model) is an interface standard for software components introduced by Microsoft in 1993. It is used to enable inter process communication and dynamic object creation in any programming language that supports this technology. The term COM is often used in the software 
development industry as a term that encompasses the OLE, ActiveX, COM+ and DCOM technologies.

The main advantage of COM is that it's a language-neutral way of implementing objects that can be used in environments different from the one they were created in. Although the interface standard has been implemented on several platforms, COM is primarily used in Microsoft Environment.

After discussion, Belsim decided to develop the Material Object in .NET C\# 2.0. The Material Object is the central point of the architecture and the link between our software and the CAPE-Open Property Package/Thermo Systems. Based on that, two Belsim software parts have been adapted to be able to create an instance of the Material Object using COM interfaces:

ValiModeller: Graphical User interface designed to model the process. This interface is developed in C++.

- ValiEngine: Calculation Engine developed in FORTRAN.

Several COM interfaces in addition to the one defined for CAPE-OPEN have been developed in the Material Object to ease the communication with the calling application.

\subsection{First Implementation}

A first CAPE-OPEN socket version 0.93 was developed in 2001 but the use of a CO-Thermo Package was very difficult for a non-expert user. The Graphical User Interface (ValiModeller) was not able to deal with a third party thermodynamic model and we had to use a text editor to define a thermo plug. Furthermore, the compliance was not complete because Vali did not use the actual compounds of the CO-Thermo Package for all the calculations. Having had this experience, eased however the development work of a CAPE-OPEN 1.0 socket.

\section{Development Phase}

\subsection{The Material Object (MO)}

The Material Object is the communication interface between the PME (Process Modeling Environment) and the PMC (Process Modeling Components). In the CAPE-OPEN 1.0 architecture, the Material Object is implemented at the PME level. In the case of our development (Thermo Socket), the Material Object was the central point of the architecture and the link between Belsim Software and Thermo Plugs making crucial this part of the system.

It was decided to develop a proprietary Material Object in C\# 2.0 using Visual Studio 2005. According the specifications of CAPE-OPEN 1.0, the Material Object developed by Belsim implements the interface 
"ICapeThermoMaterialObject". All Methods defined in this interface have been implemented.

\subsection{Cape-Open thermo plugs viewer}

The first graphical tool to be developed was a small tool based on COM technology to allow the user to see what Thermo Systems and Property Packages are available on a given computer. This tool has been developed in C\# 2.0 using Visual Studio 2005.

\subsection{Graphical user interface (ValiModeller)}

ValiModeller is the Graphical User Interface in the suite of VALI software. It is developed in $\mathrm{C}++$. When defining a validation problem you need to choose a thermodynamic package (list of compounds, list of thermodynamic methods and if you have binary parameters). In ValiModeller the option of choosing between Belsim's own thermodynamic package and a CAPE-OPEN package has been integrated.

The user needs to select between the following options:

- $\quad$ Build a thermodynamic package from existing integrated databases of compounds and Thermodynamic methods (called native thermo and compounds).

- Select a Cape-Open 1.0 Thermo Property Package available on the computer, listed in a dialog box.

ValiModeller is also able to list the compounds and properties available in the CO-Thermo Package.

The information shown in this window is collected from the Property Package through the Material Object (MO).

\subsection{Vali Engine}

In a first step, it was necessary to verify that everywhere in the code when a pure compound property is needed, the property is given by the Thermo Package (through the MO) and not by an internal calculation. These properties are: Name, Chemical Formula, CAS number, Molecular Weight, Boiling Temperature, Enthalpy of Formation.

After that, the different modules used to calculate a given property of a mixture have been modified to get the result of the calculation from the Thermo Package and not from a Vali internal routine. Here are the following properties: Liquid and Vapor Enthalpy, Volume, Entropy, Gibbs' Free Energy, Viscosity, Conductivity and Fugacities (or Fugacity Coefficients or Activity Coefficients).

The choice to perform flash calculations directly from the Thermo Package (optional) or to solve them using the native modules with the Properties obtained from the Thermo Package is also available. 


\section{Testing Phase}

Several test problems have been solved in order to test our implementation of the CAPE-OPEN 1.0 thermo socket. We selected sample flow sheets available on COCO (cape open to cape open) internet site (http://www.cocosimulator.org/index_sample.html).

R.H. Cavett [1] devised a now famous problem to test tearing, sequencing and convergence procedures of flowsheet simulation programs. This process having multiple recycle loops has been used intensively to test tearing, sequencing and convergence procedures. The flowsheet is equivalent to a four theoretical stage near isothermal distillation (rather than a conventional near isobaric type).

\subsection{Process simulation with VALI}

The process has been modelled with VALI software using its own (we call it native) thermodynamic database of chemical compounds (to define nitrogen, carbon dioxide, hydrogen sulphide, methane, ethane, propane, isobutene, nbutane, isopentane, n-pentane, n-hexane, n-heptane, n-octane, n-nonane, ndecane and n-undecane) and methods (the classical PENG-ROBINSON equation was chosen with binary parameters available on COCO internet site).

\subsection{Process simulation with VALI and CAPE-OPEN thermo plugs}

The next step was to substitute the native thermodynamic models with different imported CAPE-OPEN thermo plugs.

The first one was a "COCO Thermodynamics for Engineering Applications" called "COCO_TEA.ThermoPack.1" containing the same compounds and a modified PENG-ROBINSON equation.

The second one was a Simulis package containing the same compounds and a classical PENG-ROBINSON equation.

\subsection{Simulation results with VALI native and CAPE-OPEN thermo plugs}

The simulation results were similar with $27.34 \mathrm{kmol} / \mathrm{hr}$ in feed, $12.53 \mathrm{kmol} / \mathrm{hr}$ light products and $14.81 \mathrm{kmol} / \mathrm{hr}$ heavy products. The flashes were set at the same pressure.

The CPU time required for the different computations depends on the thermo plug type. The verification phase is speedier with the native one and much slower with the Simulis one, the same for the resolution phase.

Verification phase implies loading all the necessary data and physical property model, and checking that all required properties can be calculated.

In the resolution phase, mass and energy balance equations are solved, as well as specifications handled as pseudo measurements. 
In this case, the number of specified measurements is such that the problem is just calculable (no redundancy). The number of iteration needed to solve the problem with the prescribed accuracy differs slightly according to the thermo system used, even if the same equation of state was selected in all cases. We expect that the convergence criterion in the solution of the equation of state was not the same in all cases which may have an impact on the speed of convergence in the outer loop.

Table 1 CPU Time and Number of Iterations Comparison

\begin{tabular}{lllll}
\hline & Verification & Resolution & Total & Iterations \\
\hline Native & 0.22 & 1.1 & 2 & 3 \\
Coco & 0.37 & 3.11 & 5.11 & 3 \\
Simulis & 2.98 & 8.88 & 16 & 5 \\
\hline
\end{tabular}

\subsection{Conclusion}

Using a thermo socket is time consuming during the verification phase certainly to access the corresponding "dll" file. In this example it appears that the access of external modules to access physical properties implies a significant penalty. However, the difference can be much smaller, as we observed when using different test cases. The way the Thermoplugs are structured, and the programming language used in their development can also explain different behaviors.

Similar test has been done with HAD (hydrodealkylation) problem with similar conclusions.

\section{Conclusions}

These developments were time consuming ( 3 month part time work), and especially estimating the time needed for the development was difficult. The development required some training provided by Prosim and COLAN. Testing and debugging were also rather challenging because all the thermo plugs were not available on the same computer as our source codes.

To conclude, VALI has today a CAPE-OPEN 1.0 socket that has been successfully tested on several different CAPE-OPEN 1.0 thermo plugs. Development of an alternative socket based on the new standard 1.1 is foreseen in a near future.

\section{References}

[1] Cavett, R. H., 'Application of Numerical Methods to the Convergence of Simulated Processes Involving Recycle Loops', American Petroleum Institute, 43, 57, 1963 